British Journal of Nutrition (2016), 115, 251-261

(C) The Authors 2015. This is an Open Access article, distributed under the terms of the Creative

Commons Attribution licence (http://creativecommons.org/licenses/by/4.0/), which permits unrestricted

re-use, distribution, and reproduction in any medium, provided the original work is properly cited.

\title{
Impact of methods used to express levels of circulating fatty acids on the degree and direction of associations with blood lipids in humans
}

\author{
Susan Sergeant ${ }^{1,2}$, Ingo Ruczinski ${ }^{3}$, Priscilla Ivester ${ }^{1,4}$, Tammy C. Lee ${ }^{1,4}$, Timothy M. Morgan ${ }^{5}$, \\ Barbara J. Nicklas ${ }^{6}$, Rasika A. Mathias ${ }^{7}$ and Floyd H. Chilton ${ }^{1,4,8 *}$ \\ ${ }^{1}$ Center for Botanical Lipids and Inflammatory Disease Prevention, Wake Forest School of Medicine, Medical Center Blvd, \\ Winston-Salem, NC 27157, USA \\ ${ }^{2}$ Department of Biochemistry, Wake Forest School of Medicine, Medical Center Blvd, Winston-Salem, NC 27157, USA \\ ${ }^{3}$ Department of Biostatistics, Bloomberg School of Public Health, Johns Hopkins University, Baltimore, MD 21205, USA \\ ${ }^{4}$ Department of Physiology/Pharmacology, Wake Forest School of Medicine, Medical Center Blvd, Winston-Salem, NC 27157, USA \\ ${ }^{5}$ Department of Public Health Sciences/Biostatistical Sciences, Wake Forest School of Medicine, Medical Center Blvd, \\ Winston-Salem, NC 27157, USA \\ ${ }^{6}$ Department of Internal Medicine/Gerontology and Geriatric Medicine, Wake Forest School of Medicine, Medical Center Blvd, \\ Winston-Salem, NC 27157, USA \\ ${ }^{7}$ Department of Medicine, Division of Allergy and Clinical Immunology, The Johns Hopkins University, Baltimore, MD 21224, USA \\ ${ }^{8}$ Department of Molecular Medicine and Translational Sciences, Wake Forest School of Medicine, Medical Center Blvd, \\ Winston-Salem, NC 27157, USA
}

(Submitted 9 June 2015 - Final revision received 17 September 2015 - Accepted 5 October 2015)

\begin{abstract}
Numerous studies have examined relationships between disease biomarkers (such as blood lipids) and levels of circulating or cellular fatty acids. In such association studies, fatty acids have typically been expressed as the percentage of a particular fatty acid relative to the total fatty acids in a sample. Using two human cohorts, this study examined relationships between blood lipids (TAG, and LDL, HDL or total cholesterol) and circulating fatty acids expressed either as a percentage of total or as concentration in serum. The direction of the correlation between stearic acid, linoleic acid, dihomo- $\gamma$-linolenic acid, arachidonic acid and DHA and circulating TAG reversed when fatty acids were expressed as concentrations $v$. a percentage of total. Similar reversals were observed for these fatty acids when examining their associations with the ratio of total cholesterol:HDL-cholesterol. This reversal pattern was replicated in serum samples from both human cohorts. The correlations between blood lipids and fatty acids expressed as a percentage of total could be mathematically modelled from the concentration data. These data reveal that the different methods of expressing fatty acids lead to dissimilar correlations between blood lipids and certain fatty acids. This study raises important questions about how such reversals in association patterns impact the interpretation of numerous association studies evaluating fatty acids and their relationships with disease biomarkers or risk.
\end{abstract}

Key words: PUFA: Lipid biomarkers: Linoleic acid: Arachidonic acid

Fatty acids have diverse biological roles that are central to human health and disease. Qualitative and quantitative changes in the dietary fat content as a result of the modern Western $\operatorname{diet}^{(1-3)}$ have altered the balance of circulating and resultant tissue levels of SFA, MUFA and PUFA and their metabolites. Many of these changes have been associated with alterations in the levels of disease biomarkers and chronic inflammation, which may impact the risk for diseases such as CVD, diabetes and cancer ${ }^{(4-8)}$.

In mammals, SFA and MUFA can be obtained preformed from the diet or synthesised in vivo. In contrast, PUFA must be obtained from the diet. The two essential dietary PUFA precursors are the eighteen-carbon $n-6$ PUFA, linoleic acid (LA, C18:2n-6), and the $n$-3 PUFA, $\alpha$-linolenic acid (ALA, C18 : $3 n$-3). The long-chain PUFA can be obtained from the diet or biochemically derived from essential PUFA through a series of alternating desaturation and elongation enzymatic steps ${ }^{(9)}$. For example, the $n-6$ long-chain PUFA, arachidonic acid (ARA, C20:4n-6), can be synthesised from LA; the $n-3$ long-chain PUFA, EPA (C20:5n-3) and DPA (C22:5n-3), can be synthesised from ALA.

Abbreviations: ARA, arachidonic acid; DEMO, Diet, Exercise, Metabolism and Obesity in Older Women; LA, linoleic acid; OA, oleic acid; TC, total cholesterol. 
There remain important questions concerning the recommendations for quantities and ratios of dietary fatty acids needed to maintain health. In particular, there has been an intense debate as to whether replacing dietary SFA with the $n-6$ PUFA LA, abundant in vegetable oils, has benefited or harmed human health $^{(10,11)}$. The question of dietary requirements has been complicated by recent studies illustrating wide genetic variations in the capacity of individuals and racial/ethnic groups to metabolise dietary PUFA ${ }^{(11-13)}$

Consequently numerous studies have directly examined the relationships between levels of fatty acids in circulation or cells/ tissues and disease biomarkers and the incidence of human disease ${ }^{(14-23)}$. In such studies, fatty acid levels have been evaluated in whole blood, erythrocytes, serum, plasma or fractionated plasma components (phospholipids, cholesterol esters, TAG, unesterified fatty acids) utilising GC with flame ionisation detection. Fatty acid data from such studies typically have been expressed as a percentage of an individual fatty acid normalised to the total amount of all fatty acids measured in the sample (i.e. 100\%). This expression method can serve to overcome inter-study variations in extraction and separation efficiencies in fatty acid analyses. In contrast, a few studies have reported fatty acid levels as absolute concentrations ${ }^{(24,25)}$. This is interesting as other blood lipids and biomarkers are generally reported in units of concentration.

The current study examined associations between levels of circulating total fatty acids and blood lipids (TAG, and LDL, HDL or total cholesterol (TC)) in two human cohorts. This study has identified strong associations between circulating fatty acids and lipid biomarkers. However, these data also point out striking differences in associations garnered from data utilising the common practice of expressing fatty acids as a percentage of total $v$. data in which fatty acids are expressed as in terms of concentration $(\mathrm{mg} / \mathrm{dl}$ or $\mathrm{mmol} / \mathrm{l})$.

\section{Methods}

\section{Participants}

The Diet, Exercise, Metabolism and Obesity in older women (DEMO) study was conducted from 2003 to 2007; the details of this study have been described elsewhere ${ }^{(26)}$. The DEMO cohort consisted of ninety-three women who were overweight, but were otherwise healthy. A second, replicate, cohort was from a study designed to assess the effects of botanical and fish oils on disease biomarkers in the diabetes/metabolic syndrome subjects that was carried out from 2012 to $2013^{(27)}$. The latter diabetes/metabolic syndrome cohort was composed of fiftynine participants ( $59 \%$ women) who had been diagnosed with either early-stage type 2 diabetes or had the metabolic syndrome ${ }^{(28,29)}$. Both studies were approved by the Wake Forest School of Medicine Institutional Review Board, and all participants gave written, informed consent for their respective study as well as for future research use of their archived biospecimens. Blood lipid profiles (including TAG, and LDL, HDL or TC) were measured in plasma (DEMO) or serum (the diabetes/ metabolic syndrome study) at baseline (pre-intervention) by a qualified clinical laboratory (LabCorp).

\section{Fatty acid analysis}

Total serum fatty acids were analysed by GC with flame ionisation detection ${ }^{(30)}$ using a Hewlett Packard 5890 instrument (Agilent) with an Agilent J\&W DB-23 column $(30 \mathrm{~m}, 0.25 \mathrm{~mm}$ ID, $0 \cdot 25 \mu \mathrm{m}$ film; Agilent) fitted with an inert pre-column $(1 \mathrm{~m}$, $0.53 \mathrm{~mm}$ ID) for cool on-column injection. Fatty acid was cleaved from complex lipids and converted to methyl esters in duplicate serum samples $(100 \mu \mathrm{l})$ utilising a modification of Metcalfe et $a{ }^{(31)}$. Fatty acids in samples were identified on the basis of retention times of commercially available authentic fatty acid methyl ester standards. Triheptadecanoin $(100 \mu \mathrm{g}$; TAG of C17 : 0; Nu-Chek Prep) was included in the samples as an internal standard. Fatty acids (23-29 peaks) were routinely identified and these accounted for $>99 \%$ of the total fatty acids in the sample. Archival baseline serum samples from the DEMO study had been stored at $-80^{\circ} \mathrm{C}$ for $6-10$ years. The stability of circulating fatty acids over this time period has been reported to be excellent ${ }^{(32)}$. Fatty acid data are presented as the percentage of total fatty acids in the sample or expressed as concentration $(\mathrm{mmol} / \mathrm{l})$ in serum. Data for nineteen of the most abundant fatty acids were used for association analyses.

\section{Statistical analyses}

Measures of association between selected serum fatty acids and blood lipids are reported as Pearson's sample correlation coefficients, with statistical significance for non-zero correlations derived from hypothesis tests using Fisher's $z$-transformation. Theoretical predictions for the correlations between circulating lipids and fatty acid expressed as a percentage of total were derived using standard linear model theory and a first-order Taylor expansion for functions of multivariate random variables ${ }^{(33)}$, described in detail in the online Supplementary Materials. All analyses were carried out using the statistical environment R (http://cran.r-project.org).

\section{Results}

\section{Characteristics of the study populations}

The DEMO cohort consisted of ninety-three women $(61 \%$ European-American, 39\% African-American) ranging in age from 50 to 65 years, with an average age of 56.9 (sD 4.4) years. Aside from being overweight (BMI, 33.3 (sD 3.8$) \mathrm{kg} / \mathrm{m}^{2}$; range 26-41.3), this cohort of postmenopausal women was generally healthy, as diabetes, coronary artery disease, cancer, diseases of the liver, kidneys or lungs and tobacco use were criteria for exclusion from the study. In addition, the individuals in the cohort did not, on average, meet the criteria to be classified as having the metabolic syndrome ${ }^{(28,29)}$.

A replication cohort consisted of subjects ( $n$ 59; 59\% European-American, 39\% African-American, 2\% Asian) with the diabetes/metabolic syndrome and was composed of thirtyfive women (59\%) and twenty-four men. The average age of this cohort was 58.1 (SD 5.6) years (range 40-74) and the average BMI was $34 \cdot 2$ (sD $5 \cdot 6$ ) kg/m² (range 23-49). 


\section{Circulating fatty acid profile}

Total fatty acids were analysed in baseline fasting serum samples (before any intervention) of the ninety-three women from the DEMO cohort. A total of fifty-nine serum samples were available for analysis from the diabetes/metabolic syndrome cohort. Table 1 shows the fatty acid profile of the twenty-three to twenty-nine fatty acids routinely detected in these serum samples. These fatty acids accounted for $>99 \%$ of the fatty acids in the samples. The fatty acid data are presented using two methods of expression: the absolute concentration in $\mu \mathrm{mol} / \mathrm{l}$ of serum and the relative amount to total fatty acids (i.e. percentage of total fatty acid) in serum. Nineteen of the most abundant circulating fatty acids were subsequently used for association analyses.

\section{Relationship between selected serum fatty acids and circulating TAG levels}

Fig. 1 shows the statistically significant associations between TAG and the two most abundant circulating fatty acids, LA (31\%) and oleic acid (OA) (19-20\%), calculated as a percentage of total and as concentrations. However, the direction of the relationship between TAG and LA was negative when expressed as a percentage of total (Fig. 1(b); $r-0.53 ; P<0.0001$ ), but positive when expressed as the serum concentration (mmol/l) of LA (Fig. 1(a) $r \quad 0.60 ; P<0.0001)$. In contrast, the association between TAG and OA was positive using either fatty acid expression method ( $r 0.71$ with $P<0.0001$ for percentage of total, Fig. 1(b); and $r 0.90$ with $P<0.0001$ for absolute concentration, Fig. 1(a)).

Using an independent data set from the diabetes study cohort, we observed similar patterns (Fig. 1(c) and (d)) of relationships between LA and OA and TAG levels. Here too, the direction of the relationship between TAG and LA was negative when expressed as a percentage of total (Fig. 1(d); $r-0.53$; $P<0.0001)$, but positive when expressed as the serum concentration (mmol/l) of LA (Fig. 1(c); $r 0 \cdot 37 ; P<0 \cdot 005$ ), and the association between TAG and OA was positive using either fatty acid expression method ( $r 0.62$ with $P<0.0001$ for percentage of total, Fig. 1(d); and $r 0.87$ with $P<0.0001$ for concentration, Fig. 1(c)). We observed that the relationship between the alternative forms of fatty acid expression and TAG levels was consistent whether examining African-American and EuropeanAmerican subgroups or the entire cohort (data not shown).

To better understand the discrepancies observed with different methods of analysis, we derived a mathematical equation that could use the observed concentrations of fatty acids to predict the correlation between TAG and fatty acids when fatty acids were expressed as a percentage of total. As the percentage of total fatty acids is a non-linear function of fatty acid concentrations, we used a first-order Taylor-series expansion to estimate the correlation between TAG and fatty acid expressed as a percentage of total (see the online Supplementary Materials for mathematical details). Importantly, we observed that the variable direction in the relationship only depends on the serum fatty acid concentrations, their statistical variability and the correlation with TAG levels. Mathematically, the sign of the correlation between TAG and percentage of total solely depends on the sign of the term $\rho_{1} \sigma_{1} \mu_{2}-\rho_{2} \sigma_{2} \mu_{1}$, where $\mu_{1}$ denotes the average concentration of a serum fatty acid in the cohort, $\mu_{2}$ the average of all other fatty acid concentrations, $\sigma_{1}$ and $\sigma_{2}$ the respective standard deviations, and $\rho_{1}$ and $\rho_{2}$ the respective correlations with the TAG levels (see the online Supplementary Materials for details). Specifically, the correlation between TAG and percentage of total fatty acids will be negative if, for a given fatty acid, the product of the correlation with the TAG levels and its $\mathrm{CV}$ is smaller than the corresponding product for all other fatty acid concentrations combined:

$$
\rho_{1} \times \sigma_{1} / \mu_{1}<\rho_{2} \times \sigma_{2} / \mu_{2} .
$$

In the case of LA (Fig. 2(a)), the average concentration among the ninety-three samples (DEMO cohort) was $3.83 \mathrm{mmol} / \mathrm{l}$ (estimate for $\mu_{1}$ ), and the average of all other fatty acids was $8.27 \mathrm{mmol} / \mathrm{l}$ (estimate for $\mu_{2}$ ). The respective sD were $0.77 \mathrm{mmol} / \mathrm{l}$ (estimate for $\sigma_{1}$ ) and $2.17 \mathrm{mmol} / \mathrm{l}$ (estimate for $\sigma_{2}$ ), yielding estimated $\mathrm{CV}$ of 0.20 and 0.26 , respectively. The sample correlations with TAG levels were 0.60 (estimate for $\rho_{1}$ ) and 0.87 (estimate for $\rho_{2}$ ), respectively. As $0.12=0.60 \times 0.20<0.87 \times$ $0 \cdot 26=0 \cdot 23$, the correlation between TAG and percentage of total LA is predicted to be negative. Fig. 2(b) shows that the observed ratio of means and standard deviations (large green circle) for LA does reside in the predicted (grey) area of negative correlation.

In contrast, the average OA (Fig. 2(c)) concentration among the ninety-three samples was $2.39 \mathrm{mmol} / \mathrm{l}$, and the average of all other fatty acids was $9.70 \mathrm{mmol} / \mathrm{l}$. The respective SD were 0.76 and $2.04 \mathrm{mmol} / \mathrm{l}$, yielding estimated $\mathrm{CV}$ of 0.32 and 0.21 , respectively; the sample correlations with TAG levels were 0.90 for $\mathrm{OA}$ and 0.82 for the sum of all other fatty acids. As $0.90 \times 0.32=0.29>0.17=0.82 \times 0.21$, the predicted correlation between TAG and percentage of total OA should be positive (Fig. 2(d)). In addition, the direction of the associations of ARA (Fig. 2(e) and (f)) and DHA (Fig. 2(g) and (h)) with TAG is predicted to be negative as shown. Importantly, comparable directional relationships between these fatty acids and TAG were observed in the replication cohort (online Supplementary Fig. S1).

\section{Relationship between alternative forms of fatty acid measurement}

The mathematical model clearly established that the predicted and observed relationships between TAG and selected fatty acids (LA, OA, ARA and DHA) are in agreement. It was then important to evaluate the relationships between the alternative forms of fatty acid expression (independent of lipid biomarkers) across the wider array of fatty acids assessed in these data. As would be expected, the correlations between total and individual fatty acid concentrations in serum were all positive (not shown). In contrast, the correlation between total fatty acid concentration and the percentage of total fatty acids for an individual fatty acid were observed to be either positive or negative, depending on the fatty acid. Mathematically, the predicted sign of the correlation between total fatty acid concentration and percentage of total fatty acid depends solely on the term $\sigma_{1} \mu_{2}\left(\sigma_{1}+\rho \sigma_{2}\right)-\sigma_{2} \mu_{1}\left(\sigma_{2}+\rho \sigma_{1}\right)$, whereas, above, $\mu_{1}$ denotes the average concentration of a serum fatty acid in the population, $\mu_{2}$ the average of all other fatty acid concentrations, $\sigma_{1}$ and $\sigma_{2}$ the respective standard deviations, and $\rho$ the 


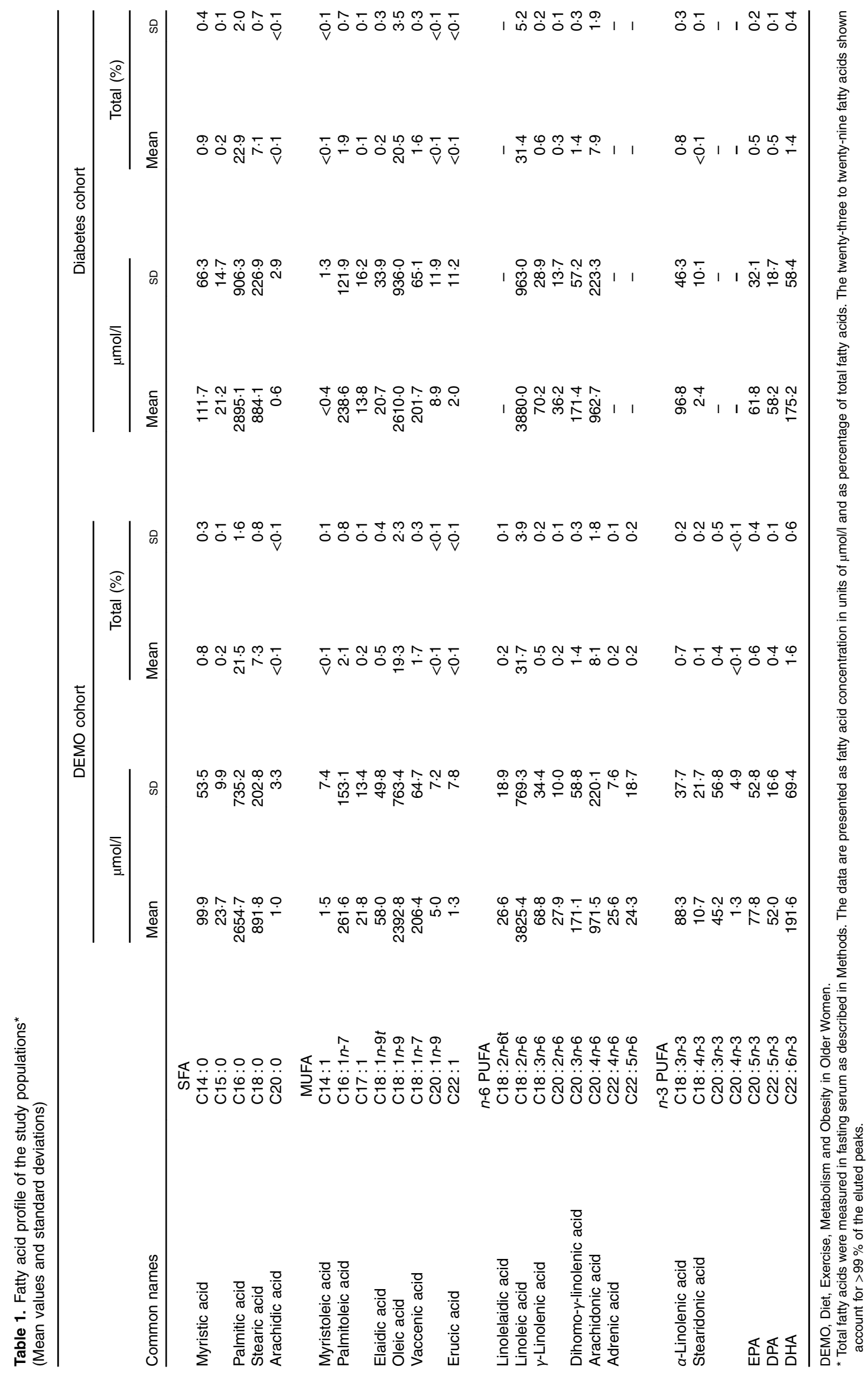


(a)

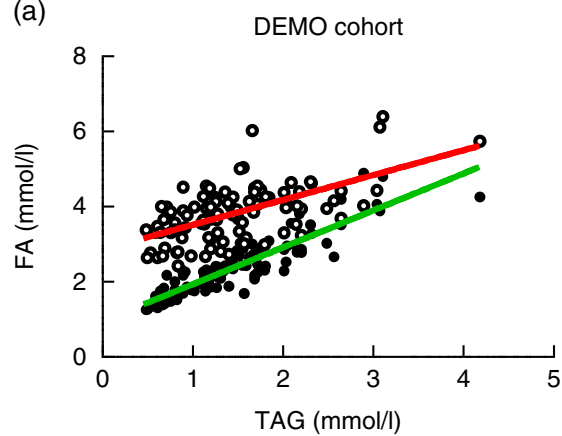

(b)

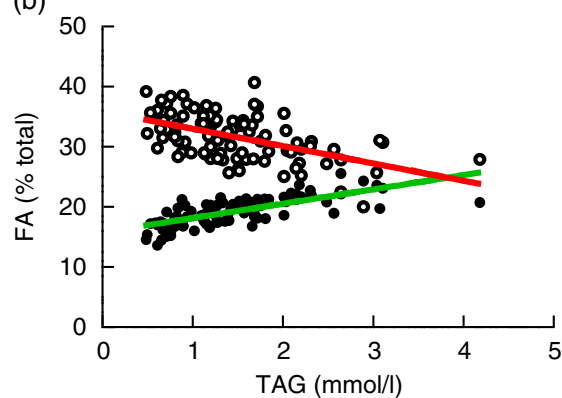

(c)

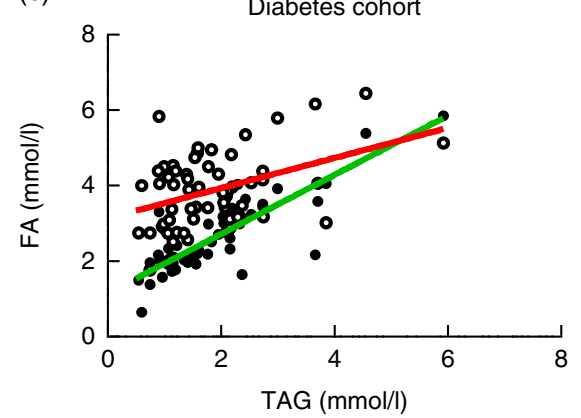

(d)

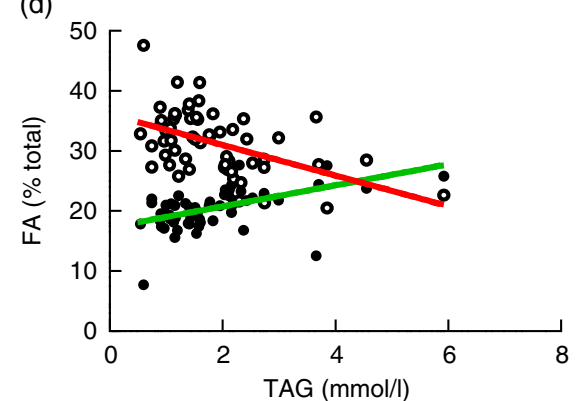

Fig. 1. Impact of fatty acid (FA) expression method on the TAG relationship with oleic acid (OA, C18:1n-9) and linoleic acid (LA, C18:2n-6). The relationship between TAG and the most abundant circulating FA, LA (31\%), differs $(\bigcirc, \mathrm{C} 18: 2 n-6)$ on the basis of the method of FA expression: concentration (mmol/l) (a); or as percentage of total (b) in the Diet, Exercise, Metabolism and Obesity in Older Women (DEMO) cohort. The same relationships were also examined in the replicate population (the diabetes/metabolic syndrome cohort; right panels) as concentration (mmol/l) (c); or as percentage of total (d). The relationship between TAG and OA (19-20\%) is unaffected $(\mathrm{C}, \mathrm{C} 18: 1 n-9)$ by the method of FA expression. For visualisation, the linear regression line is shown for each data set.

correlation between the serum fatty acid concentration and total fatty acid level less than the fatty acid of interest (see the online Supplementary Materials for details). The correlation between total fatty acid concentration and percentage of total fatty acid will be negative if, for a given fatty acid, its $\mathrm{CV}$ is small compared with the coefficient for all other fatty acid concentrations combined, with the exact threshold for the sign depending on $\rho$ :

$$
\left(\sigma_{1}+\rho \sigma_{2}\right) \sigma_{1} / \mu_{1}<\left(\sigma_{2}+\rho \sigma_{1}\right) \sigma_{2} / \mu_{2} .
$$

Among the nineteen fatty acids examined, fourteen, including OA, exhibited a positive relationship between the total fatty acid concentration and the individual fatty acid when expressed as percentage of total as evidenced by the location of the observed ratio of fatty acid means and standard deviations (Fig. 3(a); green circle). These include C18 : $1 n-9 t, \mathrm{C} 20: 5 n-3, \mathrm{C} 20: 1 n-9$, C16:1n-7, C20:3n-6, C22:5n-3, C18:3n-6, C18:3n-3, C18: $1 n-7, \mathrm{C} 17: 1, \mathrm{C} 15: 0, \mathrm{C} 14: 1, \mathrm{C} 18: 1 n-9$ and $\mathrm{C} 16: 0$, in order of increasing value of the correlation coefficient $(\rho$; range 0.32-0.94). In contrast, for five fatty acids, including LA, the correlation between percentage of total for an individual fatty acid and total fatty acid concentration was negative (Fig. 3(b)). For this group of fatty acids (C22: 6n-3, C20 : 4n-6, C20 : 2n-6, C18:2n-6 and C18:0), the observed ratio of fatty acid means and standard deviations fell in the negative correlation field. Thus, the directionality of the relationship between fatty acids as a percentage of total (a relative expression) and total fatty acid concentration (absolute expression) among fatty acid species is an apparent inherent property and independent of the TAG. Nevertheless, we believed it important to evaluate the impact of the alternative fatty acid expression methods on the relationships between fatty acids and other blood lipids, whose fatty acid content differs qualitatively and quantitatively from that in TAG.

\section{Relationship between serum fatty acid expression method and other blood lipids}

In addition to TAG, circulating fatty acids also reside in other circulating complex lipids including LDL, HDL and TC, which have been utilised as disease biomarkers. Fig. 4 summarises the associations between cholesterol-containing blood lipids and selected serum fatty acids (OA, LA, ARA, ALA, DHA) using both concentration $(\mathrm{mmol} / \mathrm{l})$ and percentage of total data. With the exception of HDL, the (Pearson's) correlations of blood lipids with fatty acid concentration (mass) were positive (blue cells), whereas the correlations with percentage of total data were either positive (blue) or negative (pink). Importantly, the mathematically predicted association values for percentage of total were nearly identical (in both magnitude and direction) to those observed for percentage of total fatty acids. As was the case for TAG, the associations of TC, LDL, HDL and the TC:HDL ratio with $\mathrm{OA}$ were consistent for both expression methods. Like LA, the long-chain $n-6$ and $n$ - 3 PUFA (ARA and DHA, respectively) showed a reversal in the direction of associations using the percentage of total fatty acid expression for TAG, TC, LDL and the ratio of TC:HDL-cholesterol. In contrast, associations with HDL tended not to undergo a change in direction between the fatty acid expression methods, except for LA, for 
(a)

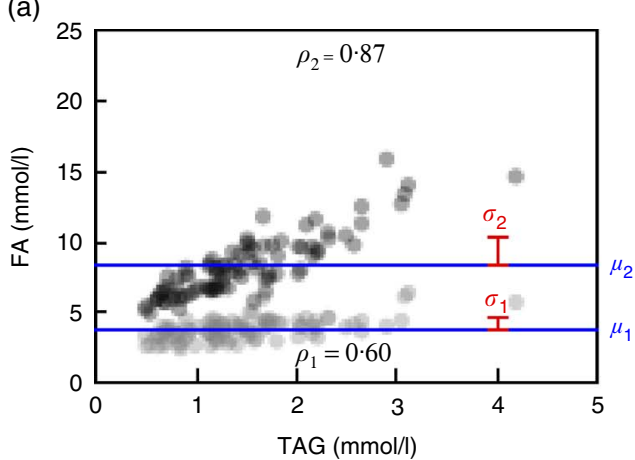

(c)

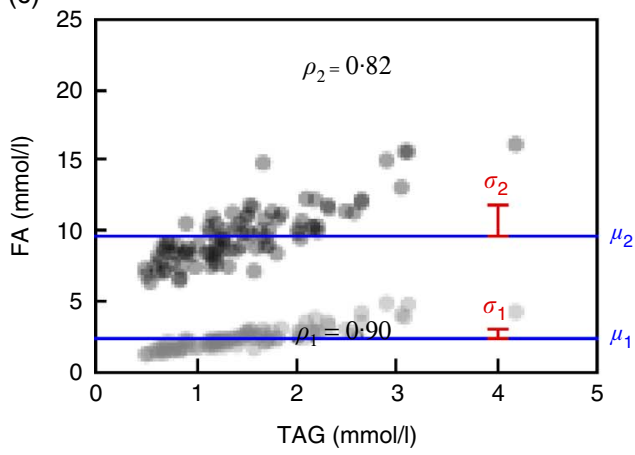

(e)

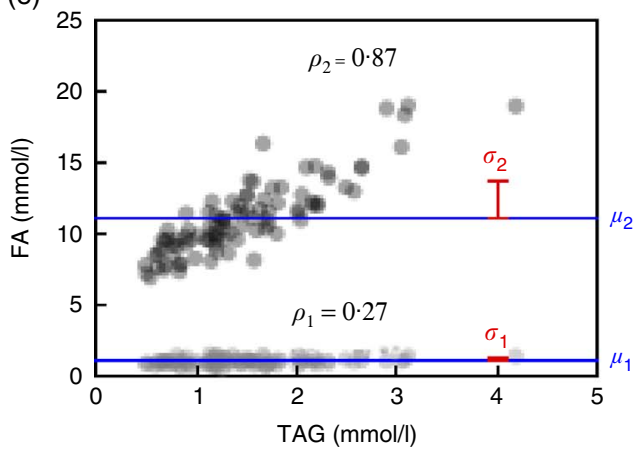

(g)

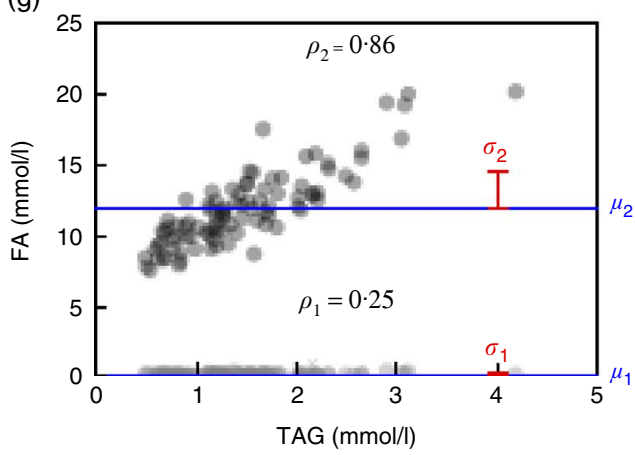

(b)

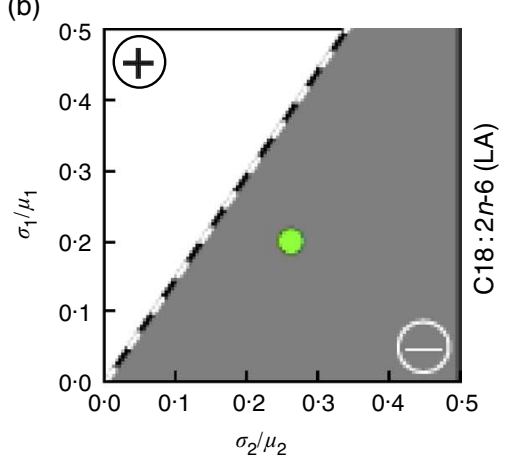

(d)

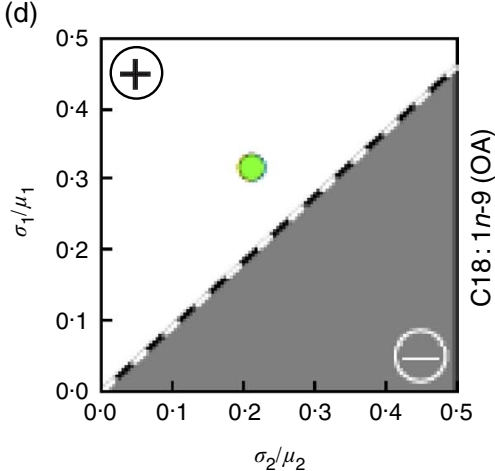

(f)

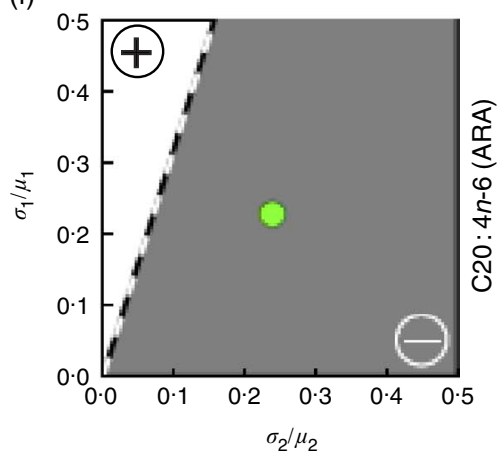

(h)

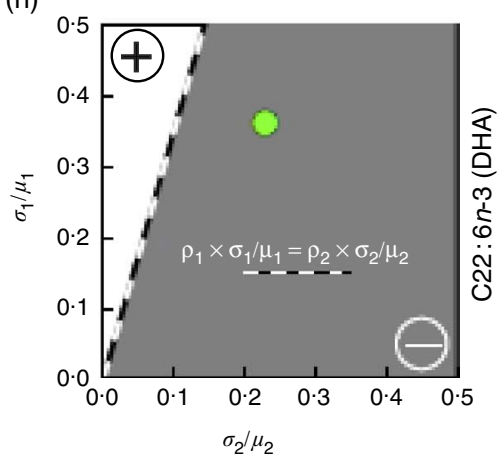

Fig. 2. Predicted TAG relationship with (OA, C18:1n-9), linoleic acid (LA, C18:2n-6), (ARA, C20:4n-6) and DHA (C22:6n-3) based on fatty acid (FA) concentration and variability (Diet, Exercise, Metabolism and Obesity in Older Women (DEMO) cohort). The TAG levels $v$. observed FA concentrations (a, c, e, g), indicating serum concentrations of $\mathrm{LA}(\mathrm{a}, \bigcirc), \mathrm{OA}(\mathrm{c}, \bigcirc)$, ARA (e, ) and DHA (,$\bigcirc)$, and total FA concentrations without $\mathrm{LA}, \mathrm{OA}, \mathrm{ARA}$ and DHA, respectively $(\bigcirc)$ ). Values are means $(\mu, \longrightarrow)$ and $\mathrm{SD}(\sigma, \mathrm{I})$, and the sample correlation with TAG $(\rho)$ is shown in the lower (LA, OA, ARA, DHA) or upper (all other FA) part of the respective scatter plots. The correlation between TAG and percentage of total FA will be negative $(\mathrm{l}, \mathrm{b}, \mathrm{d}, \mathrm{f}, \mathrm{h})$ if, for a given FA, the product of the correlation $\left(\rho_{1}\right)$ with the TAG levels and its CV $\left(\sigma_{1} / \mu_{1}\right)$ is smaller than the corresponding product for all other FA concentrations combined $\left(\rho_{2} \times \sigma_{2} / \mu_{2}\right)$. The percentage of total LA, ARA and DHA expected to be negatively correlated with TAG levels $(b, f, h)$ and the percentage of total OA expected to be positively correlated with TAG levels (d). $O$, The observed ratios of means and standard deviations. 
(a)
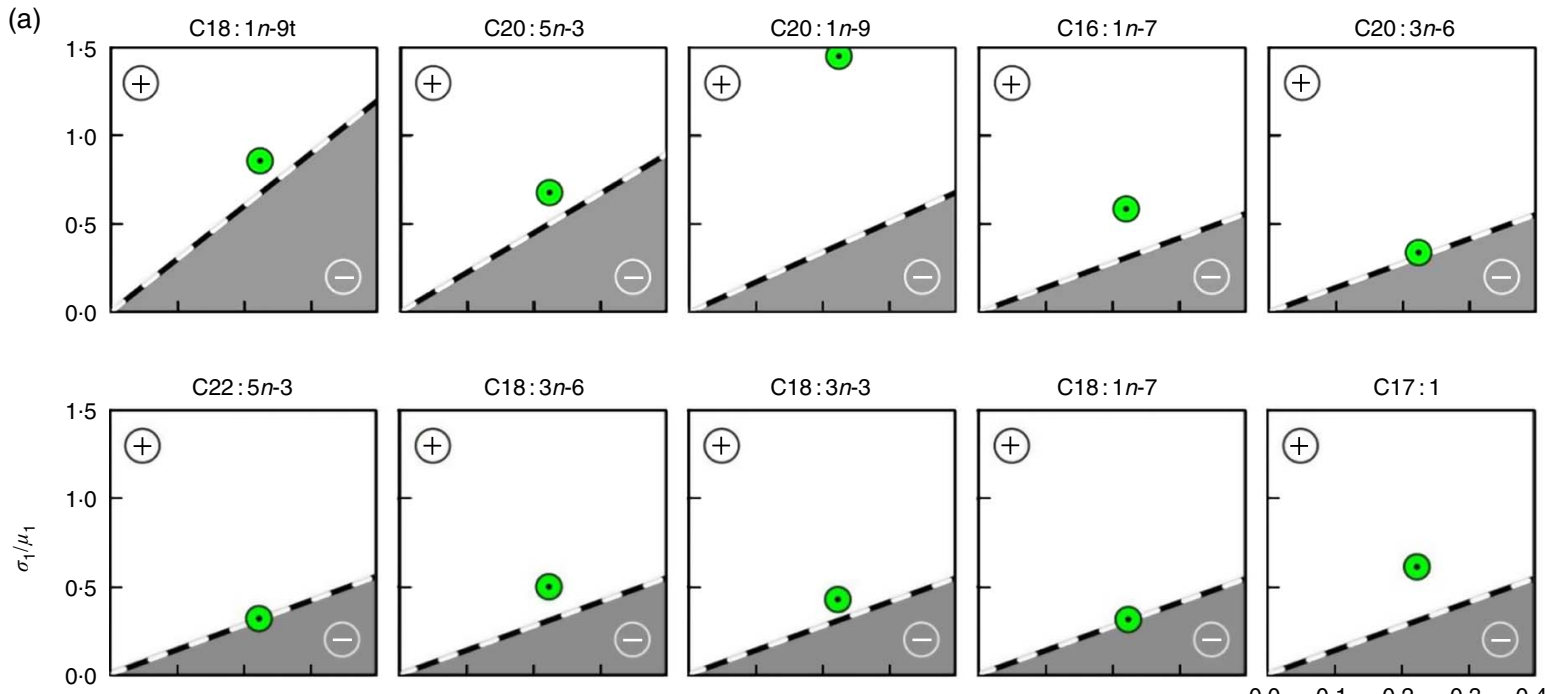

C18:3n-6
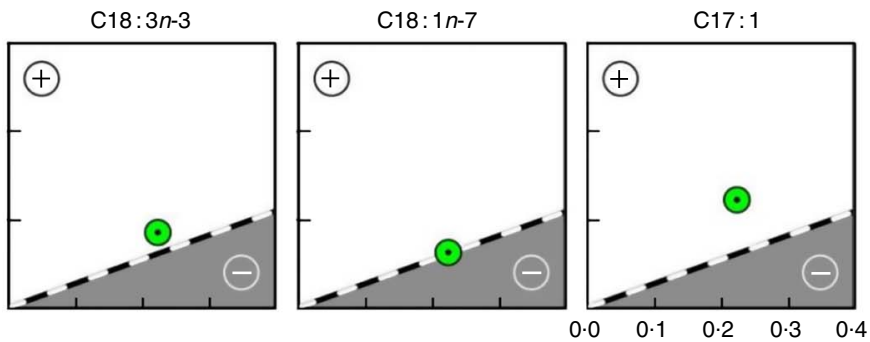

C15:0

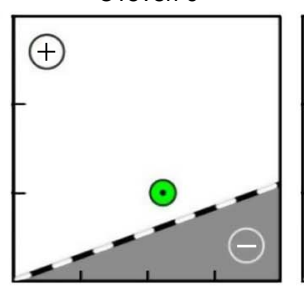

C18:1n-9

C16:0
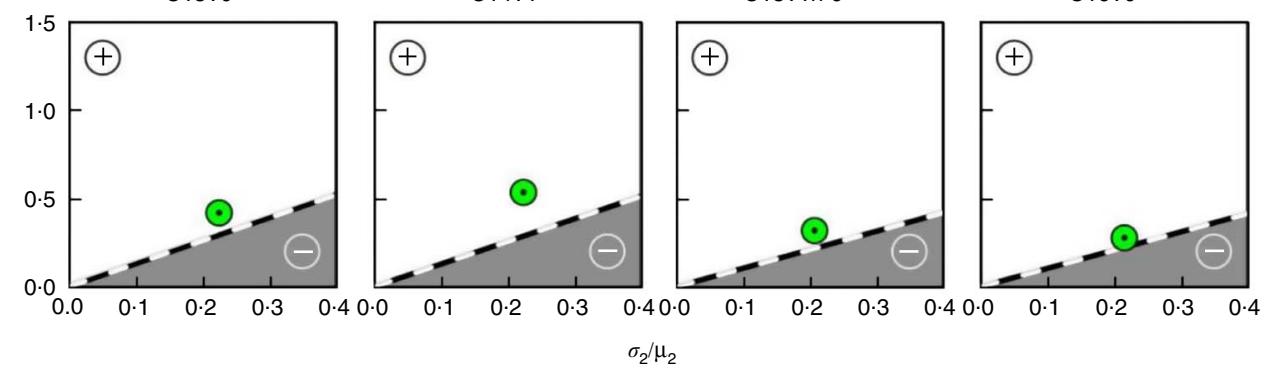

$\left(\sigma_{1}+\rho \times \sigma_{2}\right) \times \sigma_{1} / \mu_{1}$
$=$
$\left(\sigma_{2}+\rho \times \sigma_{1}\right) \times \sigma_{2} / \mu_{2}$
-

(b)

C20 : 4n-6
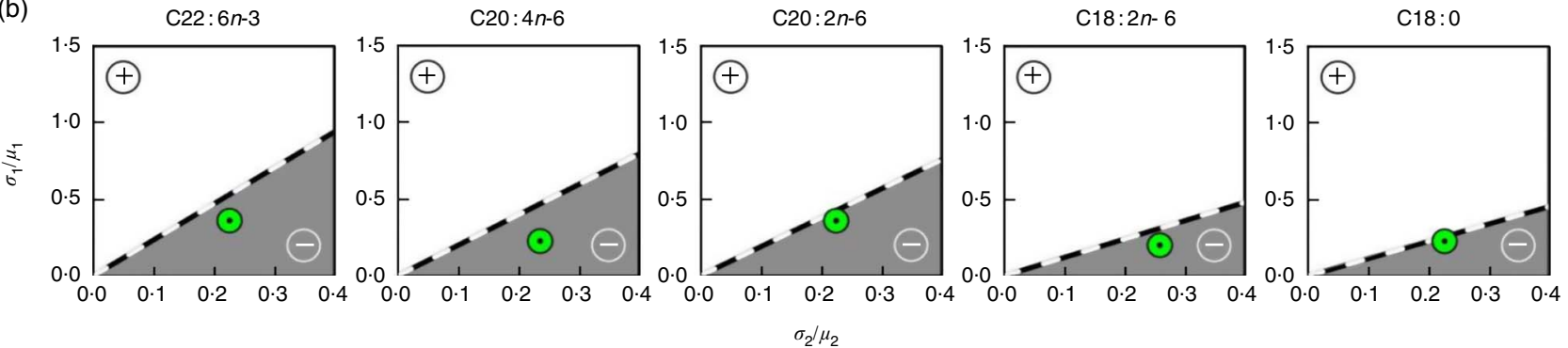

Fig. 3. Relationship of fatty acid concentration when reported as a percentage of total and total serum concentration. The expected correlations between percentage of total and total fatty acid concentration as functions of the CV $\left(\sigma_{2} / \mu_{2}\right.$ X-axis; $\sigma_{1} / \mu_{1} ; \mathrm{y}$-axis $)$ are shown. The correlation between total fatty acid concentration and percentage of total fatty acid will be negative $(\square)$ if for a given fatty acid the CV is small compared with the coefficient for all other fatty acid concentrations combined, with the exact threshold for the sign depending on the correlation $(\rho)$ between the fatty acid concentration and the sum of the concentrations of all other fatty acids. The sample standard deviations were used to predict the boundaries (- - - - ) between positive and negative correlations. (a) In all, fourteen fatty acids, including oleic acid, were positively correlated with total fatty acid concentration when expressed as a percentage of total. (b) The five fatty acids, including linoleic acid, negatively correlated with total fatty acid concentration when expressed as a percentage of total. $\odot$, The observed coefficients.

which the associations were not very robust. The associations between TAG and DHA were comparable (for mass observed, positive; percentage of total observed and predicted, negative) to that for the association between TAG and EPA+DHA (mass observed, 0.30; percentage of total observed, $-0 \cdot 21$, and predicted, -0.19). Nearly identical results, both in the robustness and direction of correlations, were observed in the replication data set (online Supplementary Fig. S2). Overall, the mathematically modelling technique appears to be useful for predicting a reversal in the direction on associations between fatty acid expression methods and blood lipids.

\section{Discussion}

The identification of relationships between fatty acids levels and disease biomarkers are important to understand potential roles of fatty acids in human disease as well as to predict disease risk or monitor disease progression. Consequently, numerous studies over the past 50 years have examined the relationships between dietary intake of fatty acids and circulating and cellular levels of fatty acid and disease biomarkers, disease incidence and progression $^{(22,23,34-40)}$. Importantly, these studies have also been the basis of dietary fatty acid recommendations for human populations ${ }^{(41,42)}$. 


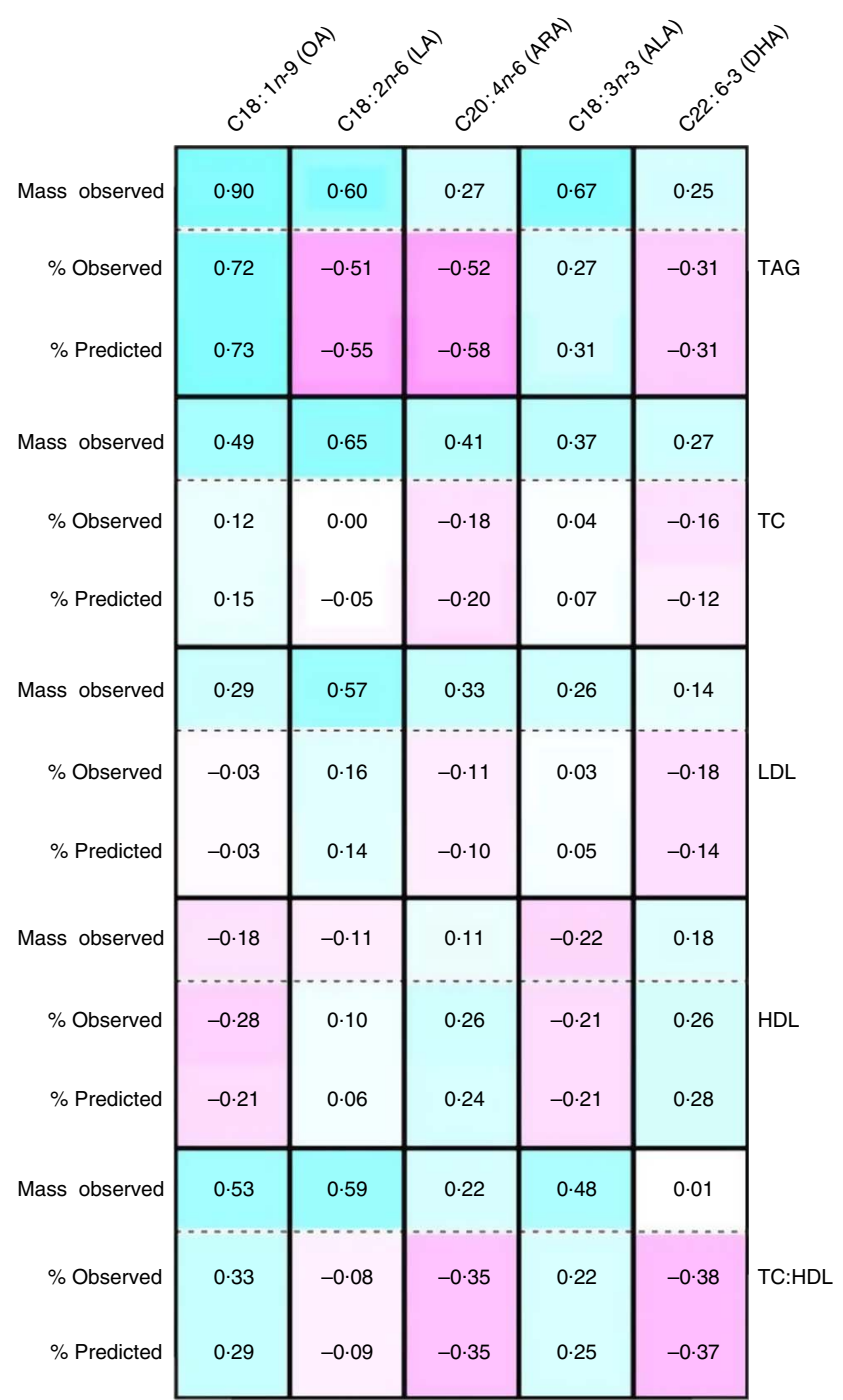

Fig. 4. Predicted relationships between blood lipids and selected PUFA (Diet, Exercise, Metabolism and Obesity in Older Women cohort). Sample correlations between cholesterol-containing blood lipids (TAG, total cholesterol (TC), LDL, $\mathrm{HDL}$, ratio of TC:HDL-cholesterol) and selected serum fatty acids (oleic acid (OA), linoleic acid (LA), arachidonic acid (ARA), $a$-linolenic acid (ALA), DHA), using both measured fatty acid concentrations ( $\mathrm{mg} / \mathrm{dl}$; 'mass observed') and percentage of total data (' $\%$ observed'). Also shown are the predicted correlations between blood lipids and percentage of total (' $\%$ predicted'). Positive $v$. negative correlations are highlighted by $\quad v$. cells, respectively.

However, numerous factors complicate the assumption that there is a link between fatty acid intake levels with disease risk or to develop dietary recommendations. For example, multiple pools of fatty acids, including both dietary fatty acids as well as endogenously synthesised fatty acids from tissues such as the liver and adipose, contribute to circulating fatty acid levels. In addition, adipose tissue is a major storage repository for fatty acids, which can be released into circulation under a variety of conditions. Moreover, recent studies show that there can be major differences in the frequencies of genetic variants that impact fatty acid levels and particularly conversion of $n-6$ and $n$-3, eighteen-carbon PUFA to long-chain highly unsaturated fatty acids in different human populations ${ }^{(12,13,43,44)}$. All of these are key factors that contribute to circulating and cellular levels of fatty acids and complicate studies that attempt to associate dietary fatty acids and disease biomarkers or risk.

In addition, fatty acids are measured in a wide variety of circulating (plasma, serum, plasma phospholipids and cholesterol esters) and cellular compartments (erythrocytes or leucocytes) or mixtures (whole blood) often without a great deal of consideration for why a particular compartment was used. Total circulating fatty acids represent a combination of free fatty acids and those in complex lipids (esterified to TAG, cholesterol esters and phospholipids) in lipoprotein particles. Each of these compartments contains different fatty acid profiles ${ }^{(45,46)}$. For example, Edelstein $^{(45)}$ showed that, in human serum, $n$-6 PUFA account for $22 \%$ of the fatty acids in the TAG, $38 \%$ in the phospholipids and $60 \%$ in the cholesterol esters. In contrast, $n$-3 PUFA accounts for only $1.8 \%$ of the fatty acids in the TAG, $3.5 \%$ in the phospholipids and $1.7 \%$ in the cholesterol esters. This issue is made even more complex given that there are hundreds of fatty acidcontaining molecular species contained within glycerolipid, phospholipid and sphingolipid classes in human plasma ${ }^{(47)}$. Therefore, results garnered from any study depend on which blood fraction or fractions are examined. Consequently, to avoid confusion and discrepancies, it is important to consider the specific hypothesis that is being addressed before determining the compartment that is to be measured. For example, there are circumstances where measurements of free fatty acids or particular complex lipid (phospholipid, glycerolipid or cholesterol ester) classes or molecular species are necessary to answer a specific question. In other circumstances, it is better to measure the plasma (serum) compartment as a whole given that specific fatty acids are selectively distributed in certain lipid classes and molecular species.

As pointed out in this study, another potential complicating factor in analysing both circulating and cellular fatty acids is the way in which fatty acid data are expressed. This latter concern has been a subject of debate for over 20 years ${ }^{(40,48,49)}$. Fatty acids have been historically expressed as a percentage of total fatty acids in a given compartment. Although this is a convenient and seemingly straightforward method of data presentation, there are mathematical considerations that must be taken into account that can impact the interpretation of the data garnered from such an analysis. The key finding of this study is that the manner in which fatty acid data were expressed (percentage of total $v$. fatty acid concentrations) has a tremendous impact on the relationship between circulating fatty acids and circulating blood lipids.

This was particularly true for $n-6$ PUFA. The literature suggests that serum LA is inversely associated with TAG, TC and LDL-cholesterol $^{(50-52)}$ when fatty acid data are expressed as a percentage of total. The percentage of total data from the DEMO cohort confirms this inverse relationship between percentage of total serum LA (Fig. 1 and 2) and TAG, but the direction of this relationship reversed when LA levels were expressed as a concentration of the fatty acid in serum (mmol/l). Similarly, inverse associations have been reported between ARA and TAG or LDL-cholesterol ${ }^{(50,52)}$, but again these are reversed when ARA is expressed as concentration. We caution though that conclusions can be drawn only for fatty acids measured here and 
their respective ranges of concentrations observed, as other relationships might exist, for example, among much higher circulating levels of DHA, or among other fatty acids.

On the basis of the derivations and findings reported in this manuscript, we also recommend that previously reached conclusions about LA and AA and their relationship to lipid profile and CHD should be re-visited. The issue of whether relationships reported using absolute $v$. relative concentrations agree or disagree is purely mathematical, and depends on the mean levels of these concentrations and their respective covariances. This paper reveals that the different methods of expressing concentrations lead to dissimilar correlations between blood lipids and some fatty acids, a phenomenon that might well be present among other biomarker relationships as well.

It is not surprising that there are positive associations between total fatty acids and individual fatty acids when expressed as concentration. It is also expected that total fatty acid concentrations would be associated with TC, LDL-cholesterol and TAG as fatty acids are esterified to cholesterol, TAG and phospholipids in circulation. These complex lipids are packaged within lipoprotein particles as major transporters of fatty acids to cells and tissues. However, in an attempt to standardise and simplify the data by using a percentage of total analysis, it does appear that there is a great potential for misinterpreting such data, especially in light of the number of highly influential papers and a recent meta-analysis that have concluded that certain fatty acids, and particularly $n$-6 PUFA, have cardio-protective attributes ${ }^{(37,41)}$

It could be argued that separating circulating fatty acids into fractions such as plasma phospholipids is a better approach and the percentage of total data would be more reliable in this context. However, this would again assume that the size of the fraction is uniform in all individuals and/or that all individual fatty acids within the fraction increased or decreased at the same rate. Taken together, these data suggest that when examining fatty acids in total serum (or plasma) or in isolated fractions such as plasma phospholipids the concentration of individual fatty acids should be measured. This method of analysis is particularly important when attempting to determine the relationships between fatty acids and disease biomarkers.

\section{Conclusion}

Associations between circulating fatty acids and blood lipids have influenced our view of fatty acid with regard to their importance in human disease and dietary recommendations. However, we show that different methods of fatty acid expression result in non-uniform relationships between certain circulating fatty acids and circulating complex lipids such as TAG, and LDL, HDL or TC. In addition, we have demonstrated that the commonly used percentage of total expression method can be mathematically modelled using fatty acid concentration data, and this provides a means of predicating a reversal in the direction of association. Thus, the method by which circulating and potentially cellular fatty acids are expressed appears to be important when interpreting relationships between fatty acid levels and blood lipid levels. These data suggest that relationships between fatty acids and other biomarkers and indices of CHD should be re-visited with this in mind.

\section{Acknowledgements}

The authors thank John Parks of Wake Forest School of Medicine for thoughtful and stimulating discussions during the preparation of this paper.

This work was supported by a grant from the National Institutes of Health, P50 AT002782 (F. H. C.)

Author contributions are as follows: F. H. C. and S. S. designed the study; S. S., F. H. C., R. A. M. and I. R. wrote the manuscript; S. S. and P. I. performed the fatty acid analyses; T. M. M. performed the statistical analysis, S. S. analysed the data; I. R. analysed and modelled the data; T. C. L. provided the diabetes study data set; and B. J. N. provided the DEMO cohort biospecimens. F. H. C. is an unpaid consultant for Gene Smart Health and receives no compensation or equity in this role. This information has been disclosed to Wake Forest University Health Sciences and outside sponsors, as appropriate, and is institutionally managed. All other authors declare no conflicts of interest.

\section{Supplementary material}

For supplementary material/s referred to in this article, please visit http://dx.doi.org/doi:10.1017/S0007114515004341

\section{References}

1. Blasbalg TL, Hibbeln JR, Ramsden CE, et al. (2011) Changes in consumption of omega- 3 and omega- 6 fatty acids in the United States during the 20th century. Am J Clin Nutr 93 , 950-962.

2. Cordain L, Eaton SB, Sebastian A, et al. (2005) Origins and evolution of the Western diet: health implications for the 21st century. Am J Clin Nutr 81, 341-354.

3. Eaton SB (1992) Humans, lipids and evolution. Lipids 27, 814-820.

4. Albert CM, Campos H, Stampfer MJ, et al. (2002) Blood levels of long-chain $n-3$ fatty acids and the risk of sudden death. $N$ Engl J Med 346, 1113-1118.

5. Lopez-Garcia E, Schulze MB, Manson JE, et al. (2004) Consumption of ( $n-3)$ fatty acids is related to plasma biomarkers of inflammation and endothelial activation in women. J Nutr 134, 1806-1811.

6. Erkkilä A, de Mello VDF, Risérus U, et al. (2008) Dietary fatty acids and cardiovascular disease: an epidemiological approach. Prog Lipid Res 47, 172-187.

7. Williams CD, Whitley BM, Hoyo C, et al. (2011) A high ratio of dietary $n-6 / n-3$ polyunsaturated fatty acids is associated with increased risk of prostate cancer. Nutr Res 31, 1-8.

8. Chajès V, Thiébaut ACM, Rotival M, et al. (2008) Association between serum trans-monounsaturated fatty acids and breast cancer risk in the E3N-EPIC study. Am J Epidemiol 167, $1312-1320$

9. Sprecher H (1981) Biochemistry of essential fatty acids. Prog Lipid Res 20, 13-22.

10. Mukhopadhyay R (2012) An essential debate: a controversy over a dietary recommendation for omega- 6 fatty acids shows no sign of resolving itself. $A S B M B$ Today 11, 14-19.

11. Mathias RA, Pani V \& Chilton FH (2014) Genetic variants in the FADS gene: implications for dietary recommendations for fatty acid intake. Curr Nutr Rep 3, 139-148.

12. Mathias R, Sergeant S, Ruczinski I, et al. (2011) The impact of FADS genetic variants on omega- 6 polyunsaturated fatty acid metabolism in African Americans. BMC Genet 12, 50. 
13. Sergeant S, Hugenschmidt CE, Rudock ME, et al. (2012) Differences in arachidonic acid levels and fatty acid desaturase (FADS) gene variants in African Americans and European Americans with diabetes or the metabolic syndrome. Br J Nutr 107, 547-555.

14. Mozaffarian D, Lemaitre RN, King IB, et al. (2011) Circulating long-chain $\omega-3$ fatty acids and incidence of congestive heart failure in older adults: the cardiovascular health study: a cohort study. Ann Intern Med 155, 160-170.

15. de Oliveira Otto MC, Wu JHY, Baylin A, et al. (2013) Circulating and dietary omega- 3 and omega- 6 polyunsaturated fatty acids and incidence of CVD in the Multi-Ethnic Study of Atherosclerosis. J Am Heart Assoc 2, e000506.

16. Dewailly E, Blanchet C, Lemieux S, et al. (2001) n-3 Fatty acids and cardiovascular disease risk factors among the Inuit of Nunavik. Am J Clin Nutr 74, 464-473.

17. Forouhi NG, Koulman A, Sharp SJ, et al. (2014) Differences in the prospective association between individual plasma phospholipid saturated fatty acids and incident type 2 diabetes: the EPIC-InterAct case-cohort study. Lancet Diabetes Endocrinol 2, 810-818.

18. Fretts AM, Mozaffarian D, Siscovick DS, et al. (2014) Plasma phospholipid saturated fatty acids and incident atrial fibrillation: the Cardiovascular Health Study. J Am Heart Assoc 3, e000889.

19. Rissanen T, Voutilainen S, Nyyssönen K, et al. (2000) Fish oil-derived fatty acids, docosahexaenoic acid and docosapentaenoic acid, and the risk of acute coronary events: the Kuopio Ischaemic Heart Disease Risk Factor Study. Circulation 102, 2677-2679.

20. Ferrucci L, Cherubini A, Bandinelli S, et al. (2006) Relationship of plasma polyunsaturated fatty acids to circulating inflammatory markers. J Clin Endocrinol Metab 91, 439-446.

21. Fernández-Real J-M, Broch M, Vendrell J, et al. (2003) Insulin resistance, inflammation, and serum fatty acid composition. Diabetes Care 26, 1362-1368.

22. Brasky TM, Darke AK, Song X, et al. (2013) Plasma phospholipid fatty acids and prostate cancer risk in the SELECT trial. J Natl Cancer Inst 105, 1132-1141.

23. Crowe FL, Allen NE, Appleby PN, et al. (2008) Fatty acid composition of plasma phospholipids and risk of prostate cancer in a case-control analysis nested within the European Prospective Investigation into Cancer and Nutrition. Am J Clin Nutr 88, 1353-1363.

24. Schwertner HA \& Mosser EL (1993) Comparison of lipid fatty acids on a concentration basis vs weight percentage basis in patients with and without coronary artery disease or diabetes. Clin Chem 39, 659-663.

25. Bradbury KE, Skeaff CM, Green TJ, et al. (2010) The serum fatty acids myristic acid and linoleic acid are better predictors of serum cholesterol concentrations when measured as molecular percentages rather than as absolute concentrations. Am J Clin Nutr 91, 398-405.

26. Nicklas BJ, Wang X, You T, et al. (2009) Effect of exercise intensity on abdominal fat loss during calorie restriction in overweight and obese postmenopausal women: a randomized, controlled trial. Am J Clin Nutr 89, 1043-1052.

27. Lee TC, Ivester P, Hester AG, et al. (2014) The impact of polyunsaturated fatty acid-based dietary supplements on disease biomarkers in a metabolic syndrome/diabetes population. Lipids Health Dis 13, 196.

28. Alberti KGMM, Eckel RH, Grundy SM, et al. (2009) Harmonizing the metabolic syndrome: a joint interim statement of the International Diabetes Federation Task Force on Epidemiology and Prevention; National Heart, Lung, and Blood Institute; American Heart Association; World Heart Federation; International
Atherosclerosis Society; and International Association for the Study of Obesity. Circulation 120, 1640-1645.

29. Grundy SM, Brewer HB, Cleeman JI, et al. (2004) Definition of metabolic syndrome: report of the National Heart, Lung, and Blood Institute/American Heart Association conference on scientific issues related to definition. Circulation 109, 433-438.

30. Weaver KL, Ivester P, Seeds MC, et al. (2009) Effect of dietary fatty acids on inflammatory gene expression in healthy humans. J Biol Chem 284, 15400-15407.

31. Metcalfe LD, Schmitz AA \& Pelka JR (1966) Rapid preparation of fatty acid esters from lipids for gas chromatographic analysis. Anal Chem 38, 514-515.

32. Matthan NR, Ip B, Resteghini N, et al. (2010) Long-term fatty acids stability in human serum cholesteryl ester, triglyceride and phospholipid fractions. J Lipid Res 51, 2826-2832.

33. Aitchison J (1986) The Statistical Analysis of Compositional Data Monographs on Statistics and Applied Probability. London: Chapman \& Hall Ltd.

34. Mensink RP, Zock PL, Kester AD, et al. (2003) Effects of dietary fatty acids and carbohydrates on the ratio of serum total to HDL cholesterol and on serum lipids and apolipoproteins: a meta-analysis of 60 controlled trials. Am J Clin Nutr 77, 1146-1155.

35. Laaksonen DE, Nyyssönen K, Niskanen L, et al. (2005) Prediction of cardiovascular mortality in middle-aged men by dietary and serum linoleic and polyunsaturated fatty acids. Arch Intern Med 165, 193-199.

36. Rizos EC, Ntzani EE, Bika E, et al. (2012) Association between omega-3 fatty acid supplementation and risk of major cardiovascular disease events: a systematic review and meta-analysis. JAMA 308, 1024-1033.

37. Johnson GH \& Fritsche K (2012) Effect of dietary linoleic acid on markers of inflammation in healthy persons: a systematic review of randomized controlled trials. J Acad Nutr Diet 112, 1029-1041.

38. Jump DB, Depner CM \& Tripathy S (2012) Omega-3 fatty acid supplementation and cardiovascular disease: thematic review series: new lipid and lipoprotein targets for the treatment of cardiometabolic diseases. J Lipid Res 53, 2525-2545.

39. Ramsden CE, Hibbeln JR, Majchrzak SF, et al. (2010) n-6 Fatty acid-specific and mixed polyunsaturate dietary interventions have different effects on CHD risk: a meta-analysis of randomised controlled trials. Br J Nutr 104, 1586-1600.

40. Schwertner HA \& Mosser EL (1994) Lipid fatty acids calculated on a concentration vs percentage basis. Am J Clin Nutr 59, 1093

41. Harris WS, Mozaffarian D, Rimm E, et al. (2009) Omega-6 fatty acids and risk for cardiovascular disease: a science advisory from the American Heart Association Nutrition Subcommittee of the Council on Nutrition, Physical Activity, and Metabolism; Council on Cardiovascular Nursing; and Council on Epidemiology and Prevention. Circulation 119, 902-907.

42. Kris-Etherton P, Fleming J \& Harris WS (2010) The debate about $n-6$ polyunsaturated fatty acid recommendations for cardiovascular health. J Am Diet Assoc 110, 201-204.

43. Tanaka T, Shen J, Abecasis GR, et al. (2009) Genome-wide association study of plasma polyunsaturated fatty acids in the InCHIANTI study. PLoS Genet 5, e1000338.

44. Mathias RA, Bickel CA, Beaty TH, et al. (2000) A study of contemporary levels and temporal trends in inbreeding in the Tangier Island, Virginia, population using pedigree data and isonymy. Am J Phys Anthropol 112, 29-38.

45. Edelstein C (1986) General properties of plasma lipoproteins and apolipoproteins. In Biochemistry and Biology of Plasma Lipoproteins, pp. 495-505 [AM Scanu and AA Spector, editors]. New York: Marcel Dekker. 
46. Spector A (2001) Plasma free fatty acid and lipoproteins as sources of polyunsaturated fatty acid for the brain. $J \mathrm{Mol}$ Neurosci 16, 159-165.

47. Quehenberger O, Armando AM, Brown AH, et al. (2010) Lipidomics reveals a remarkable diversity of lipids in human plasma. J Lipid Res 51, 3299-3305.

48. Chow CK (2009) Fatty acid composition of plasma phospholipids and risk of prostate cancer. Am J Clin Nutr 89, 1946.

49. McCulloch M, Alvarez E, Gendreau R, et al. (2014) RE: serum phospholipid fatty acids and prostate cancer risk in the SELECT trial. J Natl Cancer Inst 106, dju024.
50. Motoyama KR, Curb JD, Kadowaki T, et al. (2009) Association of serum $n-6$ and $n-3$ polyunsaturated fatty acids with lipids in 3 populations of middle-aged men. Am J Clin Nutr 90, 49-55.

51. Jelenkovic A, Bogl LH, Rose RJ, et al. (2014) Association between serum fatty acids and lipoprotein subclass profile in healthy young adults: exploring common genetic and environmental factors. Atherosclerosis 233, 394-402.

52. Choo J, Ueshima H, Curb JD, et al. (2010) Serum n-6 fatty acids and lipoprotein subclasses in middle-aged men: the population-based cross-sectional ERA-JUMP study. Am J Clin Nutr 91, 1195-1203. 